\title{
A Bell-type theorem without hidden variables
}

\author{
Henry P. Stapp \\ Lawrence Berkeley National Laboratory, \\ University of California, Berkeley, California 94720
}

\begin{abstract}
It is shown that no theory that satisfies certain premises can exclude faster-than-light influences. The premises include neither the existence of hidden variables nor counterfactual definiteness, nor any premise that effectively entails the general existence of outcomes of unperformed local measurements. All the premises are compatible with Copenhagen philosophy and the principles and predictions of relativistic quantum field theory. The present proof is contrasted with an earlier one with the same objective.
\end{abstract}




\section{INTRODUCTION}

The premises of Bell's original hidden-variable theorem ${ }^{1}$ postulate the existence of a substructure that determines in a local manner the outcomes of a set of alternative possible measurements at most one of which can actually be performed. The implementation of the locality condition in this way thus involves a technical hidden-variable assumption that goes beyond the locality condition itself. Consequently, Bell's proof of the inconsistency of this local hidden-variable assumption with certain predictions of quantum theory casts no serious doubt on the locality condition: its implementation via the assumed hidden-variable substructure would appear to be the more likely cause of the derived inconsistency.

Bell ${ }^{2}$ later introduced a seemingly weaker local hidden-variable assumption. However, this latter form can be shown ${ }^{3.4}$ to be equivalent to the original one, apart from errors that tend to zero as the number of experiments tends to infinity. Thus both forms of the hidden-variable assumption place strong conditions on the class of theories that are covered by the theorems. These conditions are logically equivalent to the assumption that values can be pre-assigned conjunctively and locally to all of the outcomes of all of the alternative possible measurements. That assumption conflicts with what I believe to be the orthodox quantum philosophical attitude that one should not make any assumption that effectively postulates the existence of a well defined outcome of a localized measurement process that is not performed. Thus these hidden-variable theorems place in no serious jeopardy the basic locality idea that a free choice made by an experimenter in one space-time region has no influence in a second region that is space-like separated from the first.

The present paper shows that this locality idea fails, however, not only under the hiddenvariable assumption (or some closely related presumption that effectively ensures the existence, within the theory, of outcomes of unperformed local measurements), but also in a much larger class of theories, namely those that are compatible with the properties of free choice and no backward-in-time influence on observed outcomes, and that yield certain predictions of quantum theory in experiments of the Hardy type..$^{\frac{5}{}}$ The first two of these three properties is now described.

Free Choices. For the purposes of understanding and applying quantum theory, the choice of which experiment is to be performed in a certain space-time region can be treated as an independent free variable localized in that region. Bohr repeatedly stressed the freedom 
of the experimenter to choose between alternative possible options. This availability of options is closely connected to his complementarity idea that the quantum state contains complementary kinds of information pertaining to the various alternative mutually exclusive experiments that might be chosen. Of course, no two mutually incompatible measurements can both be performed, and an outcome of an experiment can be specified only under the condition that that particular experiment be performed. This "free choice" assumption is important because it allows the causal part of cause-and-effect relationships to be identified: it allows the choices made by experimenters to be considered to be causes. This identification underlies all Bell-type arguments about causal relationships.

No Backward-in-Time Influence (NBITI). An outcome that has already been observed and recorded in some spacetime region at an earlier time can be considered fixed and settled, independently of which experiment a far-away experimenter will freely choose to perform at some later time. This assumption assigns no value to a local measurement except under the condition that this local measurement be performed. But any such locally observed value is asserted to be independent of which measurement will at some later time be freely chosen and performed in a spacelike separated region. This NBITI assumption is required to hold in at least one Lorentz frame of reference, henceforth called LF.

This NBITI assumption is compatible with relativistic quantum field theory. In the Tomonaga-Schwinger ${ }^{6.7}$ formulation the evolving state is defined on a forward moving spacelike surface. Their work shows that this surface can be defined in a continuum of ways without altering the predictions of the theory, so that no Lorentz frame is singled out as preferred. On the other hand, their formalism allows the quantum state to be defined by the constant time surfaces in any one single Lorentz frame that one wishes to choose, and shows that in this one frame the evolution, including all reductions associated with specific outcomes of measurements, proceeds forward in time, with a well defined past that is not influenced either by later free choices made by experimenters or by the outcomes of the later measurements. Thus this NBITI assumption is compatible with the principles and predictions of relativistic quantum field theory. (Included in this assumption is the tacit assumption that if an outcome appears to an observer, then the mutually exclusive alternative does not occur: the many-worlds idea that both outcomes occur is excluded.)

This NBITI assumption is a small part of the larger locality condition in question here, which is the demand that what an experimenter freely chooses to do in one region has no 
effect in a second region that is spacelike separated from the first. The no backward-in-time part of the no-faster-than-light condition can be imposed without generating any difficulties or conflict with relativistic quantum field theory. But a faster-than-light effect then appears elsewhere. In particular the following theorem holds:

Theorem. Suppose a theory or model is compatible with the premises:

1. Free Choices: This premise asserts that the choice made in each region as to which experiment will be performed in that region can be treated as a localized free variable.

2. No Backward in Time Influence: This premise asserts that experimental outcomes that have already occurred in an earlier region (in frame LF) can be considered to be fixed and settled independently of which experiment will be chosen and performed later in a region spacelike separated from the first.

3. Validity of Predictions of quantum theory (QT): Certain predictions of quantum theory in a Hardy-type experiment are valid.

Then this theory or model violates the following Locality Condition: The free choice made in one region as to which measurement will be performed there has, within the theory, no influence in a second region that is spacelike separated from the first.

\section{PROOF OF THE THEOREM}

The theorem refers to the following Hardy-type $e^{5}$ experimental set-up. There are two experimental spacetime regions $L$ and $R$, which are spacelike separated, with $L$ lying earlier than $R$ in $L F$. The experimenter in $L$ freely chooses either $L 1$ or $L 2$, and an outcome, either + or - , then appears in region $L$. Then the experimenter in region $R$ freely chooses either $R 1$ or $R 2$, and one or the other of the two alternative possible outcomes, + or - , then appears in $R$.

The detectors are assumed to be $100 \%$ efficient, so that for whichever measurement is chosen in $L$, one of the two alternative possible outcomes of that measurement, either + or -, will appear in $L$, with each of these possibilities occurring about half the time, and for whichever measurement is then chosen in $R$, some outcome of that measurement, either + or - , will appear in $R$. 
For each of the two choices $L 1$ or $L 2$ available to the experimenter in $L$, and for each of the two alternative possible outcomes + or - of that experiment, quantum theory makes predictions for both of the two alternative choices $R 1$ and $R 2$ available to the experimenter in $R$.

In the statements that follow, the symbol $L 1$ will be an abbreviation of the statement "Experiment $L 1$ is performed in $L$." The symbols $L 2, R 1$, and $R 2$ will have analogous meanings.

The symbol $L 1+$ will stand for the assertion, "Experiment $L 1$ is performed in $L$ and outcome + of that experiment appears in $L . "$ The symbols $L 1-, L 2+, L 2-, R 1+, R 1-$, $R 2+$, and $R 2-$ have analogous meanings. Using these abbreviations the first two pertinent predictions of QT for this Hardy setup are that: ${ }^{8}$ Under the condition that $L 2$ is performed in $L$,

$$
\text { If } R 2+\text {, then } L 2+
$$

and

$$
\text { If }(L 2+\text { and } R 1) \text { then } R 1-\text {. }
$$

If, in accordance with our assumption, the choice made in $R$ does not affect the outcome that has already occurred in $L$, then these two conditions entail:

Property 1. Quantum theory predicts that if an experiment of the Hardy-type is performed then, $L 2$ implies $S R$, where,

$$
\begin{array}{r}
S R=\text { If } R 2 \text { is performed and gives outcome }+ \text {, then if, instead, } \\
\qquad 1 \text { had been performed the outcome would have been }- \text {. }
\end{array}
$$

Proof of Property 1. The concept "instead" is given an unambiguous meaning by the combination of the premises of "free choice," and "no backward in time influence;" the choice between $R 1$ and $R 2$ is to be treated, within the theory, as a free variable, and switching between $R 1$ and $R 2$ is required to leave any outcome in the earlier region $L$ undisturbed. But then statements (1) and (2) can be joined in tandem to give the result $S R$.

The second two pertinent predictions of QT for this Hardy setup are

Under the condition that $L 1$ is performed in $L$,

$$
\text { If }(L 1-\text { and } R 2) \text { then } R 2+
$$


and

$$
\text { If }(L 1-\text { and } R 1) \text { then sometimes } R 1+\text {. }
$$

If our premises are valid, then these two predictions entail:

Property 2. Quantum theory predicts that if an experiment of the Hardy-type is performed then: " $L 1$ implies $S R$ " is false.

\section{Proof of Property 2:}

Quantum theory predicts that if $L 1$ is performed, then outcome - appears about half the time. Thus if $L 1$ is chosen, then there are cases where $L 1-$ is true. But in a case where $L 1$ - is true, the prediction (3) asserts that the premise of $S R$ is true. But statement (4), in conjunction with our two premises that give meaning to "instead," implies that the conclusion of $S R$ is not true: if $R 1$ is performed instead of $R 2$, the outcome is not necessarily $R 1-$, as it was in case $L 2$. So there are cases where $L 1$ is true but $S R$ is false.

The conclusion is that in any theory or model in which the three assumptions (free choices, NBITI, certain predictions of quantum theory) are valid, the statement $S R$ must be always true if the free choice in region $L$ is $L 2$, but must sometimes be false if that free choice in $L$ is $L 1$. But the truth or falsity of $S R$ is defined by conditions on the truth or falsity of statements describing possible events located in region $R$. The fact that the truth of $S R$ depends in this way on a free choice made in region $L$, which is space-like-separated from region $R$, can reasonably be said to represent the existence within that theory or model of some sort of faster-than-light influence. This conclusion is discussed in the following sections.

\section{HIDDEN VARIABLES AND COUNTERFACTUAL ARGUMENTS}

The previous argument rests heavily on the use of counterfactuals: the key statement $S R$ involves, in a situation in which $R 2$ is performed and gives outcome "+," the idea "if, instead, $R 1$ had been performed ..." The Bell-type hidden-variable assumptions also are essentially counterfactual in nature. That is because the hidden variables identify instances in which the outcomes of all four of the alternative possible experiments can be considered simultaneously. But this similarity does not mean that the three assumtions used here are logically equivalent to the Bell-type hidden-variable assumptions. The Bell-type assumptions, in their original 
deterministic hidden-variable form, clearly entail the three assumptions used here. But these three assumptions do not entail the Bell-type hidden-variable assumptions. Indeed, our assumptions are, as repeatedly emphasized, compatible with orthodox Tomonaga-Schwinger relativistic quantum field theory, whereas the assumptions of Bell, either in its original form, or in terms of the factorization property assumed in the later probabilistic form of Bell's assumption,$\stackrel{2}{2}$ are in direct logical conflict with the principles of relativistic quantum field theory. Thus the assumptions made here are logically weaker than those of the traditional Bell's theorems.

\section{CONNECTION TO EARLIER WORK}

The aim of this work is similiar to that of an earlier work of this author $\underline{\underline{9}}$ That work was criticized by Unruh,,$\underline{\underline{10}}$ Mermin,$\underline{11}$ and Shimony and Stein $\underline{12}$ on various grounds. I have answered these objections $\frac{13,14.15}{1}$ However, the very existence of those challenges shows that the approach used in Ref. 9 has serious problems, which originate in the fact that it is based on classical modal logic.

Classical modal logic provides the possibility of a concise logically rigorous proof based on an established logic. However, that virtue is overshadowed by the following drawbacks:

1. Although the symbolic proof is concise and austere, that brevity is based on a background that most physicists lack, which means that most physicists cannot fully understand it without a significant investment of time.

2. The question arises as to whether the use of classical modal logic begs the question by perhaps being based in implicit ways on the deterministic notions of classical physics.

3. Classical modal logic itself is somewhat of an open question, and it is not immediately clear to what extent these issues undermine the proof.

For these reasons I have in the present formulation relied only on quantum thinking and language throughout: there is no appeal to concepts unfamiliar to physicists.

But the present proof differs from the 1997 version by more than just the use of the language of physicists. The earlier proof introduced the assumption LOC2. That assumption was introduce in order to set up a reducio ad absurdum argument: it was meant to be proved 
false. But a lot of the discussion in my earlier augument was whether I had adequately justified this "assumption," which, however, I was trying to prove false. The present version gives a straightforward proof of the key properties 1 and 2, without introducing the false assumption LOC2.

A subtle objection to the earlier argument was made by Shimony and Stein $\frac{12}{}$ : Although the explicit condition for the truth of $S R$ is specified entirely by the truth or falsity of statements about possible events localized in region $R$ - and hence the proven dependence of the truth of $S R$ upon which experiment is freely chosen in $L$ seems explictly to require an influence in $R$ of that choice made in $L$ - the word "instead" that occurs in $S R$ harbors, they say, an implicit dependence of $S R$ on the choice made in $L$, and that the implicit dependence upon $L$ upsets the conclusion.

I believe this objection to be invalid. The word "instead" gets its meaning from the explicitly stated assumption that the choices made by the experimenters can be treated as free variables, coupled with the explicitly stated assumption that, under any condition chosen by the experimenter in the earlier region $L$, whatever he/she observes in that region can be considered to be independent of the choice freely made in the later region $R$ by the other observer. These explicitly stated conditions are what enter into the logical structure of the proof, which then entails that the truth value of at least one statement whose truth or falsity is specified in terms of the outcome of a possible experiment in region $R$ must depend non-trivially on the choice made by the experimenter in the spacelike separated region $L$. This conclusion represents some sort of failure of the notion that no influence of any kind can act over a spacelike interval.

\section{CONNECTION TO THE BOHR-EINSTEIN DEBATE}

The conclusion obtained here about faster-than-light influences parallels and complements Bohr's reply to the paper of Einstein, Podolsky, and Rosen $\stackrel{\underline{\underline{16}}}{\text { That discussion was }}$ not directly about "faster-than-light" influences: it was explicitly about whether actions

performed on one system can influence "real" properties of another system. In the gedanken experiments described by EPR, the action of one experimenter could be made in one place, whereas the property in question could be measured far away at the same time. So the issue was implicitly about the possibility of faster-than-light influences of nearby actions on 
faraway properties.

The EPR assumption was thus, essentially, that there was no faster-than-light influence of any kind. Bohr's response ${ }^{17}$ was subtle. Rosenfeld's commentary ${ }^{18}$ on Bohr's response makes it clear that Bohr's effort to compose a response was not an easy endeavor.

The EPR argument rests strongly on counterfactual ideas, and it stresses at the end that "Indeed, one would not arrive at our conclusion if one insisted that two or more physical quantities can be regarded as simultaneous elements of reality only when they can be simultaneously measured or predicted." 16 (Italics are in the original.) But in spite of this crucial use of counterfactual ideas by EPR, Bohr did not attack their argument on those grounds. I think it likely that Bohr did not want to take the difficult road of trying to ban all use of counterfactual concepts in physics. Indeed, I believe he recognized that counterfactual concepts do play an important role in the pragmatic approach to physics that he was pursuing. The core of Bohr's response was to insist that there was no faster-than-light "mechanical disturbance," but to note that "there is an influence on the very conditions that define the possible types of predictions regarding the future behavior of the system."

A "mechanical disturbance" would be one capable of transmitting a signal. The EPR argument does not prove, or attempt to prove, the existence of any nonlocal mechanical influence of this kind. Their argument pertains to more subtle kinds of considerations that involve contemplating relationships between experiments that cannot be performed simultaneously, but that have outcomes that can be predicted with certainty (that is, with probability unity), and with what ensues from trying to impose upon these considerations also the idea that any predicted property that can be considered "real" in one region cannot depend upon what a faraway experimenter freely chooses to do. The argument of EPR is therefore of the same general kind as the one given here. On the other hand, the EPR argument is very different from arguments of the Bell type, because the existence of hiddenvariables was a putative consequence of the EPR argument, whereas it is an assumption of the Bell-type local hidden-variable (or local realism) theorems.

Bohr challenged not the counterfactual aspect of the EPR argument, but rather the strong form of the locality assumption assumed by EPR: he claimed that "there is an influence (of the nearby choice) upon the very conditions that define the possible types of predictions regarding the future behavior of the (faraway) system."

That statement by Bohr, the core of his reply to EPR, has puzzled many philosophers and 
physicists, and Bohr himself 19 recognized its difficulty. The present argument can be considered to be an effort to further explicate the nature of the subtle quantum non-locality that Bohr apparently recognized, and whose existence falsified, in his opinion, the EPR argument for the incompleteness of quantum theory, that is, falsified, within the conceptual framework of Copenhagen quantum theory, the EPR argument for the existence of local hidden variables of the kind postulated in the Bell local hidden-variable (or local-realism) theorems. The present theorem separates the locality aspect of the Bell local-realism postulate from the reality aspect by imposing a valid (according to Copenhagen thinking) (effectively oneway) locality condition that is expressed exclusively in terms of observables, and that is true in relativistic quantum field theory, and then deducing the failure of a no-faster-than-lightinfluence condition in the other directions, rather than by proving the failure of a strong, and very alien to quantum thinking, property that intricately combines the locality condition with a "realism" assumption that conflicts with quantum philosophy.

This argument has been carried out in a purely theoretical plane. It rests on a highly idealized theoretical conception of the experimental conditions and on purely theoretical assumptions. No attempt is made here to derive conclusions directly from empirical data, as certain approaches to Bell's theorem seek to do. A recent discussion of some of the difficulties that ensue if one tries to argue directly from experimental data is given in Ref. 20.

\section{APPENDIX A: APPENDIX: CONNECTION TO HARDY'S NOTATION}

To obtain these four predictions from Hardy's paper, $\frac{5}{\underline{\underline{5}}}$ one transforms my notation into Hardy's using

$$
\begin{aligned}
& L 1(+,-) \rightarrow\left(c_{1}, d_{1}\right) \\
& L 2(+,-) \rightarrow\left(v_{1}, u_{1}\right) \\
& R 1(+,-) \rightarrow\left(d_{2}, c_{2}\right) \\
& R 2(+,-) \rightarrow\left(u_{2}, v_{2}\right)
\end{aligned}
$$

and uses the three zero's connecting my pairs of states $(R 2+, L 2-),(L 2+, R 1+)$, and $(L 1-, R 2-)$ that arise from his Eq. (13), respectively to obtain my statements (1), (2), and (3), respectively, and uses his Eq. (13.d), which says that my matrix element $(L 1-, R 1+)$ is positive, to obtain my statement (2.4). 


\section{ACKNOWLEDGMENTS}

Extensive discussions with Asher Peres, Philippe Eberhard, Jerry Finkelstein, and particularly Abner Shimony, have contributed importantly to the development of this paper. This work was supported in part by the Director, Office of Science, Office of High Energy and Nuclear Physics, of the U.S. Department of Energy under Contract DE-AC03-76SF00098. [xx need titles of articles! $\mathrm{xx}$ ]

1 J. S. Bell, "On the Einstein-Podolsky-Rosen paradox," Physics 1, 195-200 (1964); J. Clauser and A. Shimony, "Bell's theorem: Experimental tests and implications," Rep. Prog. Phys. 41, 1881-1927 (1978).

2 J. S. Bell, Speakable and Unspeakable in Quantum Mechanics (Cambridge Univ. Press, 1987), Chap. 4.

3 H. Stapp,"Non Local Character of Quantum Theory." Epistemological Letters, June 1978. (Assoc. F. Gonseth, Case Postal 1081, Bienne, Switzerland).

4 A. Fine, "Hidden variables, joint probabilities and Bell inequalities," Phys. Rev. Lett. 48, 291$295(1982)$.

5 L. Hardy," Nonlocality for two particles without inequalities for almost all entangled states," Phys. Rev. Lett. 71, 1665-1668 (1993); P. Kwiat, P. Eberhard, A. Steinberg, and R. Chiao, "Proposal for a loophole-free Bell inequality experiment," Phys. Rev. A49, 3209-3220 (1994).

6 S. Tomonaga, "On a relativistically invariant formulation of the quantum theory of wave fields," Prog. Theor. Phys. 1, 27-42 (1946).

7 J. Schwinger, "The theory of quantized fiels, I," Phys. Rev. 82, 914-927 (1951).

8 To obtain these four predictions from Hardy's paper, one transforms my notation into Hardy's as explained in the Appendix.

9 H. P. Stapp, "Nonlocal character of quantum theory," Am. J. Phys. 65, 300-304 (1997).

10 W. Unruh, "Nonlocality, counterfactuals, and quantum physics." Phys. Rev. A 59, 126-130 (1999).

11 N. D. Mermin, Nonlocal character of quantum theory?" Am. J. Phys. 66, 920-924 (1998).

12 A. Shimony and H. Stein, "Comment on 'Nonlocal character of quantum theory' by Henry P. 
Stapp," Amer. J. Phys. 69, 848-853 (2001).

13 H. P. Stapp, "Reply to Unruh," quant-ph 9712043.

14 H. P. Stapp, "Meaning of counterfactual statements in quantum physics." Am. J. Phys. 66, 924-926 (1998).

15 H. P. Stapp, "Response to 'Comment on "Nonlocal character of quantum theory," by Henry P. Stapp,'” Am. J. Phys. 69, 854-859 (1998).

16 A. Einstein, B. Podolsky, and N. Rosen, "Can quantum mechanical description of physical reality be considered complete?" Phys. Rev.47, 777-780 (1935).

17 N. Bohr, "Can quantum mechanical description of physical reality be considered complete?" Phys. Rev. 48, 696-702 (1935).

18 A. Rosenfeld, "Commentsry of Rosenfeld (1967)," in Quantum Theory and Measurement, edited by J. A. Wheeler and W. H. Zurek (Princeton University Press, Princeton, 1983), p. 142.

19 N. Bohr, "Discussion with Einstein," in Albert Einstein:Philosopher-Scientist, edited by P. A. Schlipp (Tudor, New York, 1951), p. 234.

20 R. D. Gill, in Proceedings of the 2002 Vaxjo Conference on the foundations of quantum mechanics and probability, quant-ph/0301059. 